\title{
Breastfeeding as A Method of Health Promotion
}

\author{
Karen F Phillips* \\ *Department of Nursing, William Paterson University, Wayne, USA
}

*Corresponding author: Karen F Phillips, Department of Nursing, William Paterson University, Wayne, USA

\begin{abstract}
The literature depicts many health benefits for both mother and newborn associated with breastfeeding. Although breastfeeding initiation rates have met the goals of Healthy People, the United States lags behind the achievement of optimal exclusive breastfeeding continuation and duration rates. Billions of health care dollars could be saved along with hundreds of lives with improved breastfeeding rates. The purpose of this article was to examine the association between disease prevention, health promotion, and breastfeeding rates. The findings indicated that breastfeeding does offer some maternal and newborn protection against certain diseases and conditions which may be dose dependent, although some diseases and conditions reported mixed reviews and will require additional research. For the newborn, reduced rates of certain respiratory and gastrointestinal have been reported. Maternal benefits include a reduced rate of hypertension, ovarian, and breast cancer. Although breastfeeding rates are increasing, healthcare providers should continue to utilize multiple strategies to continue to improve breastfeeding rates including education about the association between breastfeeding and improved maternal and newborn health outcomes, the importance of breastfeeding support, workplace breastfeeding support, and community resources.
\end{abstract}

Keywords: Breastfeeding; Health Promotion; Breastfeeding; Breastfeeding Benefits; Breastfeeding Continuation; Breastfeeding Duration

\section{Introduction}

Health promotion is defined as an opportunity for individuals to take control of their own health [1]. Health promotion can involve numerous types of interventions but should always be directed at prevention of ill health [1]. Research suggests an association between breastfeeding and health promotion [2] and supports the importance of breastfeeding relative to disease prevention and health promotion. Breastfeeding contributes to disease prevention, health promotion, and saves healthcare dollars because breastfed babies are healthier overall [3,4]. Maintaining breastfeeding has been noted to be an important health preventive measure [5]. According to Healthy People [4], improving the well-being of mothers, infants, and children is a crucial public health goal because their health and well-being influences not only their future health but may also portray future public health issues in the community. The Healthy People Initiative is a collaborative effort between government agencies and professional organizations directed at improving the health of all Americans [4]. The goals of Healthy People focused on two broad areas: the elimination of health disparities and an increase in the quality and years of a healthy life [4].
Breastfeeding has been strongly associated with improved short and long-term health [6]. Breastfeeding is widely recognized as the optimum method of infant feeding and should be considered the "normative standard" of infant feeding $[1,3,7]$. Breastfeeding has been associated with a decrease in healthcare costs across the United States [7]. In fact, lower rates of breastfeeding have increased maternal and newborn health care costs to over three billion dollars a year [7]. Based on a cost analysis completed in 2005, if exclusive breastfeeding rates were at $90 \%$ for 6 months, $\$ 13$ billion dollars would be saved annually along with over 900 lives [8]. Current exclusive breastfeeding rates based on the 2015 Breastfeeding Report Card for 6 months are 24.9\%, closer to the Healthy People goals $[4,9]$. Breastfeeding initiation rates are now $83.2 \%$ and have exceeded the goals set by Healthy People 2020 [4,9].

\section{Review of the Literature}

A review of the literature depicts both maternal and newborn benefits relative to breastfeeding. A reduced risk of numerous health conditions both for the mother and newborn have been associated with breastfeeding [7]. There is increasing evidence that 
supports the risks of not breastfeeding on future health chronic conditions [5]. Health outcomes are substantially different for infants of mothers who do not breastfeed [10]. Research suggests breastfeeding as a modifiable factor that could reduce health risks in both the mother and newborn [10]. In their Position Statement on Breastfeeding, the AAP [3] indicated that studies have shown that breastfeeding results in improved health outcomes for the mother and the newborn. Dieterich et al. [11] reported that breastfeeding reduces the maternal disease burden and saves infant lives. Anatolitou [12] also reported improved maternal and newborn health outcomes stemmed from breastfeeding.

\section{Infant/Newborn Health Outcomes}

Breastfeeding provides both short and long-term benefits to infants and newborns even after breastfeeding has been discontinued [13]. Newborn benefits that have been reported are reduced risk of asthma, obesity, otitis media, respiratory, and gastrointestinal infections $[2,7,14]$. Breastfeeding has been found to play a key role in the development of the infants immune system [10]. This is significant given that diarrhea, acute respiratory infections, and fever have been reported to be major causes of mortality in children less than 5 years old [15]. A study by Khan and Islam [15] reviewed data on 1918 infants, less than 6 months old, from 2007-2014 from the Bangladesh Demographic and Health Survey. Comparisons were made among the groups and adverse outcomes related to diarrhea fever, and acute respiratory infection. When exclusive breastfeeding was discontinued at less than 6 months, the risk for diarrhea, fever, and acute respiratory infection was increased compared to exclusive breastfeeding at 6 months [15]. This study noted that $27.37 \%$ cases of diarrhea, $8.94 \%$ cases of acute respiratory infection, and $13.24 \%$ of cases of fever could be prevented if newborns were exclusively breastfed to 6 months [15]. Similarly, a study by Duijts et al. [14] from the Netherlands compared infants never breastfed to those breastfed exclusively to 4 months as well as to infants exclusively breastfed between 7 to 12 months. Lower risk for gastrointestinal infections and respiratory infections were noted among infants exclusively breastfed and partially breastfed for 4 months which continued until 6 months of age [14]. In infants breastfed exclusively for 6 months or longer, tended to have greater protection against gastrointestinal and respiratory infections than those breastfed only to 4 months [14]. This suggests that strategies should be promoted that encourage exclusive breastfeeding for at least 4-6 months [14]. Additionally, a 5- year prospective cohort study was conducted in Turkey that included 418 infants born between January and December of 2011 [16]. The purpose was to determine if there was an association between exclusive breastfeeding duration and infectious diseases as well as to examine long-term protective effects of breast milk [16]. Although this study did not report a significant decrease in respiratory infections as seen in other studies, a reduction in respiratory conditions was noted in infants breastfed 12 months or longer [16]. A significant decrease in otitis media and gastrointestinal conditions was reported in infants breastfed for 12 months or more [16]. This suggests an association between breastfeeding duration and reduction in common infectious conditions. Several studies have examined the impact of breastfeeding on childhood obesity [12]. The World Health Organization [17] reported that evidence exists that breastfeeding offers some protection against obesity. The literature depicts a $15-30 \%$ reduction in obesity with any breastfeeding, in observations through childhood and adulthood [12] although this has been noted to be controversial [11]. Maternal obesity, lifestyle, feeding practices may influence the development of obesity in children [11]. A study in Canada included 81, 226 children [18]. Height, weight, breastfeeding status during first 5 months of life along with maternal diabetes status was studied in pre-school children 4-6 years of age [18]. Data was collected from 2005 to 2013. Findings indicated that rates of overweight and obesity were lower in children who were breastfed than those who were not with the exception of children that were large for gestational age (LGA) or gestational diabetes [18].

\section{Maternal Health Outcomes}

Breastfeeding provides benefits for mothers not only during breastfeeding, but long-term benefits have been reported after breastfeeding has been discontinued [19]. Formula feeding or premature weaning from breastfeeding has been associated with increased health risks for the mother [10]. Maternal health benefits include a reduced risk of high blood pressure, Type 2 diabetes, breast, and ovarian cancer [2,7]. A reduction in the risk of stroke has also been noted in postmenopausal women who breastfed although more research is needed [20]. The literature suggests that exclusive breastfeeding for longer durations offers both short and long-term maternal health protection [11].

A study of women with live born infants in the Danish National Birth Cohort (1996-2002) examined how the effects of any, partial, and exclusive breastfeeding were associated with hypertension and cardiovascular disease [21]. Additionally, the study looked at whether prepregnancy BMI and waist circumference 7 years postpartum effected these associations [21]. A longer duration of breastfeeding was associated with a reduced risk of hypertension. Kirkegaard et al. reported any breastfeeding for greater than 4 months was linked to a reduced risk of $20-30 \%$ for development of hypertension and cardiovascular disease [21]. Study findings also included that continuation of partial breastfeeding after exclusive breastfeeding contributed to a lower rate of hypertension and cardiovascular disease although more studies are needed to further explain the link between breastfeeding and reduced rates of cardiovascular disease [21].

Jacobsen et al. [20] completed research based on data from the Women's Health Initiative Observational Study, which examined the relationship between breastfeeding and stroke risk. The study included 80, 191 participants from 40 centers in 24 states and the District of Columbia. Women were recruited between 1993-1998 and follow up continued through 2010. After adjustment for non-modifiable confounders, the findings showed that any breastfeeding was associated with a lower risk of stroke [20]. This was found to be strongest for non-Hispanic Blacks. According to Jacobsen et al. [20], a longer duration of breastfeeding was associated with a lower risk of stroke in all women studied and among non-Hispanic whites and non-Hispanic blacks. The study concluded that there was an association, which included a dose response between breastfeeding and a 
lower risk of stroke among postmenopausal women. This was noted to be significant even after adjustments were made for stroke risk factors and lifestyle variables [21]. This could be an important finding given the fact that stroke is the fourth leading cause of death in women [22].

Some earlier studies reported limited evidence to support an association between breastfeeding and reduced risk of ovarian cancer. A study in China from 2006-2008 examined the association between breastfeeding and ovarian cancer [23]. There were 493 women with ovarian cancer compared to 472 women in the control group. Data collected included pregnancy history, livebirths, number of children breastfed, as well as the duration of breastfeeding. Face- to- face interviews were completed with family members in attendance to verify patient recall. In this study, the number of children breastfed, and duration of breastfeeding was found to have a reduced risk of ovarian cancer [23].

Schwarz and Nothnagle [24], who reviewed 30 case-controlled studies and 5 cohort studies, reported women who never breastfed were $32 \%$ more likely to be diagnosed with ovarian cancer.

Breast cancer researchers have examined the effects of breastfeeding duration and the risk of development of breast cancer with mixed results. A review of 18 studies, 1 pooled study, and 3 meta-analyses was completed by the Continuing Update Project [CUP] Panel of the American Institute for Cancer Research [AICR] [25]. The dose response meta- analysis showed significant decreased risk of pre and postmenopausal breast cancer with increased breastfeeding duration [25]. The longer a woman breastfeeds, the lower the risk. Other studies showed a decreased risk but was not as significant [25]. The committee concluded that breastfeeding probably protects against breast cancer [25]. Islami et al. [26] reviewed 27 studies and concluded that any breastfeeding was associated with a $10 \%$ lower risk of breast cancer in tumors that were negative for estrogen and progesterone compared to women who did not breastfeed. However, those with positive estrogen and progesterone did not depict any significance. More research is needed on breastfeeding and positive receptor breast cancers to determine if any association exists [26]. A meta-analysis of 47 studies by Schwarz and Nothnagle [24] concluded that breast cancer rates decrease by more than $4 \%$ per year that a woman breastfeeds in her lifetime. Schwarz and Nothnagle noted that women with the BRAC 1 gene saw a 37\% decrease in breast cancer risk [24].

\section{Conclusion}

The literature suggests an association between not breastfeeding and an increase in adverse maternal and newborn health outcomes [10]. Breastfeeding affords the best opportunity for optimal newborn/ infant health outcomes [3]. WHO reports breastfeeding to be one of the most "effective ways of improving child health and survival." [27]. The CDC supports breastfeeding as the best nutritional source for infants [7]. A cost analysis by Bartick and Reinhold [8] indicated that $\$ 13$ billion and over 900 lives, primarily infant and newborn, could be saved annually if we were able to attain a $90 \%$ exclusive breastfeeding rate for 6 months in the United States. The continuation of suboptimal breastfeeding rates will amass billions of dollars as well as hundreds of preventable deaths [8]. Great strides have been made in improving breastfeeding rates, particularly exclusive breastfeeding rates, but there is room for improvement. Although breastfeeding initiation rates are high at $83.2 \%$, exclusive breastfeeding rates at 3 months are $46.9 \%$ and at 6 months are $24.9 \%$ [9]. While these rates have increased, they have not increased significantly between 2014-2015 [9].

The high initiation rates suggest that mothers do want to breastfeed [9]. Numerous factors could impact continuation rates including lack of education and support, early return to work, lack of workplace breastfeeding practices that support breastfeeding women among others [9]. Establishing and maintaining practices that offer education and support are critical in improving breastfeeding rates [28]. Support and guidance during early breastfeeding experiences are crucial to prolonged breastfeeding [28]. Breastfeeding women need education and support from healthcare providers, as well as society as a whole, to encourage and promote exclusive breastfeeding to reduce the risks of adverse health outcomes reported in formula fed infants $[9,11]$. Healthcare practices should be reviewed to support and promote breastfeeding in all phases to improve long-term breastfeeding continuation. Interventions leading to improved health outcomes should include the benefits of breastfeeding as well as the reported association between breastfeeding and adverse health outcomes [11]

\section{References}

1. World Health Organization [WHO] (2019) What is health promotion?.

2. Ip S, Chung M, Raman G, Trikalinos TA, Lau J (2009) A summary of the Agency for Healthcare Research and Quality's evidence report on breastfeeding in developed countries. Breastfeeding Medicine 4(suppl 1): S17-S30.

3. (2012) Breastfeeding and the use of human milk. American Academy of Pediatrics [AAP].

4. Healthy People 2020 (2019) Maternal, Infant, and Child Health.

5. (2007) A call to action on breastfeeding-a fundamental public health issue. American Public Health Association [APHA].

6. (2008) Achieving exclusive breastfeeding in the United States: Findings and recommendations. United States Breastfeeding Committee [USBC].

7. (2019) About breastfeeding: Why it matters. Centers for Disease Control [CDC].

8. Bartick M, Reinhold A (2010) The burden of suboptimal breastfeeding in the United States: A pediatric cost analysis. Pediatrics 125(5): 10481056 .

9. (2018) CDC 2018 breastfeeding report card. Centers for Disease Control [CDC].

10. Steube A (2009) The risks of not breastfeeding for mothers and infants. Reviews in Obstetrics and Gynecology 2(4): 221-231.

11. Dieterich C, Felice J, O Sullivan E, Rasmussen K (2013) Breastfeeding and health outcomes for mother-infant dyad. Pediatric Clinic of North America 60(1): 31-48.

12. Anatolitou F (2012) Human milk benefits and breastfeeding. Journal of Pediatric and Neonatal Individualized Medicine 1(1): 11-18.

13. Schanler R (2019) Infant benefits of breastfeeding

14. Duijts L, Jaddoe VW, Hofman A, Moll HA (2010) Prolonged and exclusive breastfeeding reduces the risk of infectious diseases in infancy. Pediatrics 126(1) 
15. Khan MN, Islam MM (2017) Effect of exclusive breastfeeding on selected adverse health and nutritional outcomes: A nationally representative study. BMC Public Health 17(1): 889.

16. Ardıç C, Yavuz E (2018) Effect of breastfeeding on common pediatric infections: a 5-year prospective cohort study. Arch Argent Pediatr 116(2): 126-132.

17. World Health Organization [WHO] (2014) Exclusive breastfeeding to reduce the risk of childhood overweight and obesity.

18. Kaul P, Bowker SL, Savu A, Donovan LE, Ryan EA (2018) Association between maternal diabetes, being large for gestational age, and breastfeeding on being overweight or obese in childhood. Diabetologia 62(2): 249-258.

19. Schanler R (2019) Maternal and economic benefits of breastfeeding.

20. Jacobsen L, Hade E, Collins T, Margolis K, Waring M et al. (2018) Breastfeeding history and the risk of stroke among parous postmenopausal women in the Women's Health Initiative. Journal of the American Heart Association 7(17): e008739.

21. Kirkegaard H, Bliddal M, Stovring H, Rasmussen K, Gunderson E, et al. (2018) Breastfeeding and later maternal risk of hypertension and cardiovascular disease- The role of overall and abdominal obesity. Preventive medicine 114: 140-148.

22. (2015) Leading causes of death (LCOD) in females. Centers for Disease Control [CDC], United States.

23. Su D, Pascalich M, Lee A, Binns C (2013) Ovarian cancer risk is reduced by prolonged lactation: A case control study in southern China. The American Journal of Clinical Nutrition 97(2): 354-359.

24. Schwarz E, Nothnagle M (2015) The maternal health benefits of breastfeeding. American Family Physician 91(9): 603-604.

25. American Institute for Cancer Research (2017) Diet, nutrition, physical activity and breast cancer. Continuous update project: Analyzing research on cancer prevention and survival.

26. Islami F, Liu Y, Jemal A, Zhou J, Weiderpass E, et al. (2015) Breastfeeding and breast cancer risk by receptor status- A systematic review and meta-analysis. Annals of Oncology 26(12): 2398-2407.

27. World Health Organization [WHO] (2017) Ten facts on breastfeeding.

28. Haroon S, Das J, Salam R, Imdad A, Bhutta Z (2013) Breastfeeding promotion interventions and breastfeeding practices: A systematic review. BMC Public Health 13 (3): 3-20.

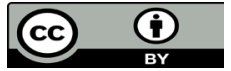

This work is licensed under Creative Commons Attribution 4.0 License

To Submit Your Article Click Here:

Submit Article

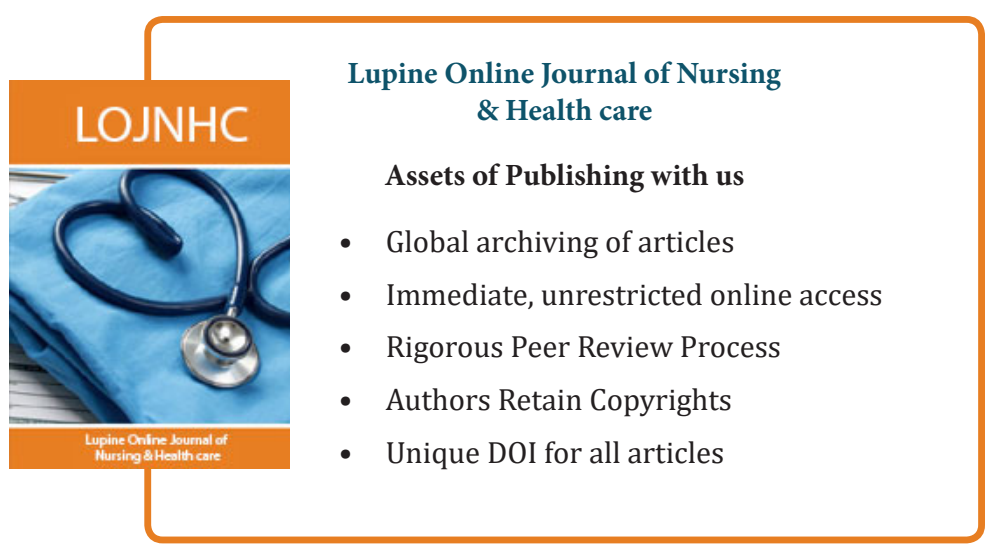

